\title{
Image Segmentation Using Truncated Compound Normal with Gamma Mixture Model
}

\author{
Viziananda Row Sanapala ${ }^{1}$, Sreenivasa Rao Kraleti ${ }^{2}$, Srinivasa Rao Peri ${ }^{3}$ \\ ${ }^{1}$ Associate Professor, Dept of Computer Science \& Systems Engg, Andhra University, \\ Visakhapatnam, India \\ ${ }^{2}$ Professor, Dept of Statistics, Andhra University, Visakhapatnam, India \\ ${ }^{3}$ Professor, Dept of Computer Science \& Systems Engg, Andhra University, Visakhapatnam, India
}

\begin{abstract}
In this paper, we formally present the truncated compound normal with gamma distribution model and define a mixture model(TCNGM) based on this as an extension work to the proposed compound normal with gamma mixture(CNGM) model introduced by us in our earlier work on image segmentation. We present update equations for this model for maximum likelihood estimation (MLE) procedure under Expectation Maximization (EM) framework, construct EM algorithm, and test the feasibility of the model to solve mixture density estimation problem in general and image segmentation in particular. We have found this model to be a competing one in the context of variations in data distributions within probabilistic framework.
\end{abstract}

Keywords: Probabilistic models, Compound Distributions, Mixture Distributions, Truncated Distributions, Maximum Likelihood Estimation, EM Framework.

Abbreviations: CNGM, GM, NM, EM, MLE, TCNGM

\section{INTRODUCTION}

In our earlier work [1],[2], image segmentation problem as mixture density estimation problem has been formally described for the compound normal with gamma mixture model (CNGM) and its truncated version (TCNGM). Mathematical expressions for maximum likelihood estimates of model parameters $\mu_{l}, c_{l}$, and $v_{l}$ have been derived. In this paper, we present image segmentation using EM framework for computing updated parameter estimates as it was done for compound normal with gamma mixture model [1],[2]. In this and the following section, we present the background information in some detail. In Section 3.2, EM algorithm for the proposed model is constructed. In Section 3.3, implementation and results produced are detailed. We place our concluding remarks in Section 4.

\subsection{Introduction to Image Segmentation}

Early stages of image analysis include edge detection and image segmentation whose solutions are mostly driven by intensity value discontinuity and similarity in the local neighborhood. Edge detection techniques look for intensity value discontinuity or abrupt changes in intensity value, based on which the image is partitioned into a set of edges. It is based on the use of some threshold, around which intensity value discontinuity occurs [3],[4],[5].

Segmentation methods use similarity property to detect distinct objects, each of which having similar properties for attributes like intensity value, texture, and others in a given image. In other words, the goal of image segmentation in this context is to detect region homogeneity or similarity in the local neighborhood and extend this to the entire image based on different methods like thresholding, region growing, and other approaches. Thus, it results in a segment whose said attributes take similar values in it. A segmentation method, given a user specified number of segments, generates distinct segments, each of which satisfying the above mentioned goal. Region segmentation is considered more useful than edge detection since regions contain more information than edges. Thus, it has been still actively pursued by research community in general [6].

However, image segmentation is an ill posed problem because it may be subjective or objective within the perspective of the end application. For example, homogeneous regions can be easily 
separated and thus segmentation here is more objective and is easily accomplished as is done for synthetic images or for certain images produced in medical imaging. But, the more general problem of image segmentation involving natural images is more subjective since for natural images region homogeneity is not well defined due to natural and environmental reasons [6],[7]. Image segmentation finds its applications in more than one domain such as content based image retrieval, machine vision, object detection, medical imaging, video surveillance, and several other areas.

\subsection{Image Segmentation Approaches}

Image segmentation may be thought of as a kind of the more general notion of clustering that partitions data into groups based on some similarity criterion used to delineate groups. Further, clustering is different from classification in that no a priori knowledge about class label (training data) is used. In clustering, the data is grouped based on the principle of maximizing the intraclass similarity and minimizing the interclass similarity [8].There exist a number of clustering approaches based on how the data is modeled. For example, hierarchical clustering uses connectivity models, Kmeans algorithm uses centroid models, clique problem uses graph theoretic models, EM algorithm uses distribution models, and other approaches use context specific models.

According to Tianhu Lei, and Jayaram K. Udupa [6], image segmentation approaches may broadly be classified into two based on how the image data is viewed; 1) a statistical model based approach that is used to describe the distribution of the data and its constituent components and 2) a graph theoretic approach in which structural organization of pixels and their interconnectedness in the local neighborhood is the basis for describing the image as a set of image regions [6],[9].

\section{Probabilistic Model Driven Approach}

In probability and statistics, a probability distribution assigns a probability to each outcome of a random experiment. A probability distribution can either be univariate or multivariate. A univariate distribution gives the probabilities of a single random variable taking on various alternative values; a multivariate distribution is a joint probability distribution that gives the probabilities of a set of two or more random variables(dimensions) taking on combinations of values[10]. In the following subsections, the theory related to probabilistic model driven approach and the corresponding mixture density estimation problem is introduced since it forms the basis for the proposed study of the image segmentation problem as mixture density estimation problem.

\subsection{Normal Distribution}

A great many of the techniques used in applied statistics are based upon the normal distribution. A random variable $X$ is defined to be normally distributed in the continuous domain if its density is given by

$f_{x}(x)=f_{x}(x ; \mu, \sigma)=\frac{1}{\sigma \sqrt{2 \pi}} e^{-(x-\mu)^{2} / 2 \sigma^{2}}$

where the parameters $\mu$ (location or mean) and $\sigma$ (standard deviation) satisfy $-\infty<\mu<\infty$ and $\sigma>0$. Any distribution defined by a density function given above is called a normal distribution. If random variable $X$ is normally distributed with mean $\mu$ and variance $\sigma^{2}$, we will write $X \sim N\left(\mu, \sigma^{2}\right)$. A standard normal distribution is defined by setting mean to 0 and variance to 1 , and we will write $X \sim N(0,1)$. Usually a normal distribution is identified by a bell shaped curve as shown in Fig. 1 (green in color).

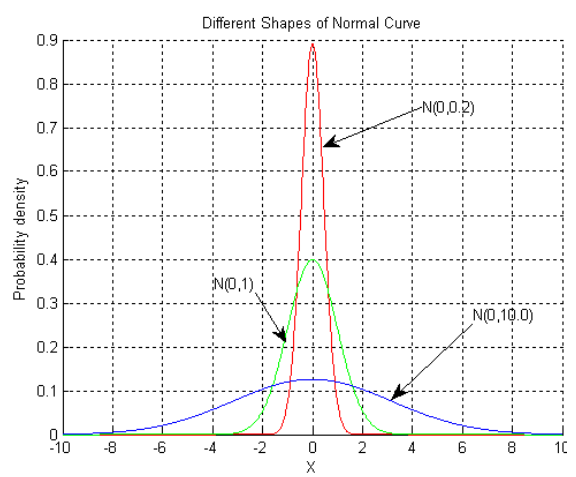

Figure1. Normal Densities $X \sim N\left(\mu, \sigma^{2}\right)$ 


\subsection{Gamma Distribution}

A random variable $X$ is defined to be following gamma distribution if its density is given by

$f_{x}(x)=f_{x}(x ; r, \lambda)=\frac{\lambda}{\Gamma(r)}(\lambda x)^{r-1} e^{-\lambda x}$

where $0 \leq x<\infty, r>0$, and $\lambda>0$. Here, $r$ and $\lambda$ are known as shape and rate (inverse of scale) parameters respectively. $\Gamma($.$) is the gamma function. If r=1$, gamma density specializes to exponential density[10]. Figure 2 shows different shapes of gamma curves for $\lambda=1$.

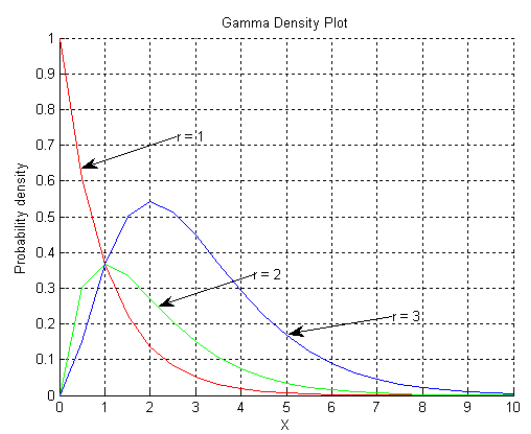

Figure2. Gamma Densities $(\lambda=1)$

\subsection{Compound Distributions}

As stated by S. C. Gupta and V. K. Kapoor in [11], consider a random variable $X$, whose distribution depends on a single parameter $\theta$ which instead of being regarded as fixed constant, is also a random variable following a particular distribution. In this case, we say that the random variable $X$ has a compound or composed distribution.

\subsection{Compound Normal with Gamma Distribution}

As given in [12] by Normal L. Johnson et al, a compound normal with gamma distribution or $\operatorname{Normal}\left(\mu, \sigma^{2}\right){ }_{\sigma^{-2}}^{\Lambda} \operatorname{Gamma}\left(c \chi_{v}^{2}\right)$ is formed by ascribing a distribution to $\sigma^{2}$ i.e., variance by considering it as a random variable and fitting a new distribution. The corresponding distribution is defined to have a density function given as

$f(x)=(2 c)^{\frac{-v}{2}}\left[\Gamma\left(\frac{v}{2}\right)\right]^{-1} \int_{0}^{\infty}[\sqrt{2 \pi} \sigma]^{-1}\left(\sigma^{-2}\right)^{\left(\frac{v}{2}-1\right)} . \exp \left[-\left(2 c \sigma^{2}\right)^{-1}\left(2 \sigma^{2}\right)^{-1}(x-\mu)^{2}\right] d \sigma^{-2}$

After some mathematical transformations and further treatment, Equation (1.3) reduces to

$f(x)=\frac{1}{c^{1 / 2} B(1 / 2, v / 2)}\left[1+\frac{(x-\mu)^{2}}{c}\right]^{-(v+1) / 2}$

where $\mu, c, v$ are location, scale, and shape parameters. The compound normal with gamma distribution model that has been introduced now forms the basis for our work and a mixture model for this has been used used to solve the image segmentation problem[1][2].

\subsection{Truncated Distributions}

In statistics, a truncated distribution is a conditional distribution that results from restricting the domain of some other probability distribution. Truncated distributions arise in practical statistics in cases where the ability to record, or even to know about, occurrences is limited to values which lie above or below a given threshold or within a specified range [10].In general, if $X$ is a random variable with density $f_{x}($.$) and cumulative distribution F_{x}($.$) , then the density of X$ truncated on the left at $a$ and on the right at $b$ is given by

$\frac{f_{x}(x)}{F_{x}(b)-F_{x}(a)}$

For example, image segmentation problem may be viewed as mixture density estimation problem and since gray level images are spatially represented using an eight bit intensity or pixel value, the pixels only take values ranging between 0 and 255 , each representing a particular gray value ranging between black and white. This strongly suggests to define a truncated mixture model, with $0 \leq x \leq$ 255 in place of the more general case of $-\infty<x<+\infty$ for the random variable $x$ that represents 
intensity value, for image segmentation because truncated distributions model finite range data well in comparison to the more general model.

\subsection{Mixture Distribution}

A brief introduction as given by Mood et al in [10] to the concept of contagious distribution or a mixture is given here. If $f_{0}(),. f_{1}(),. \ldots, f_{n}(),. \ldots$ is a sequence of density functions which are either all discrete density functions or all probability density functions which may or may not depend on parameters, and $p_{0}, p_{1}, \ldots, p_{n}, \ldots$ is a sequence of parameters satisfying $p_{i} \geq 0$ and $\sum_{i=0}^{\infty} p_{i}=1$, then $\sum_{i=0}^{\infty} p_{i} f_{i}(x)$ is a density function, which is sometimes called contagious distribution or a mixture.For example, the current literature on statistical image segmentation techniques mostly assumes the data describing the image as a mixture of component distributions, as shown in Fig. 3[6],[13],[14],[15] .

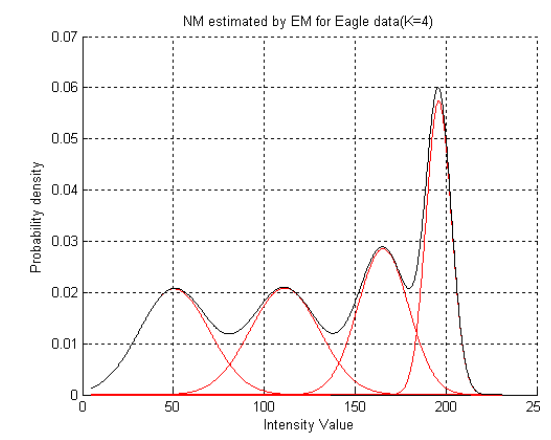

Figure3. An Example Mixture Distribution

\subsection{Clustering as Mixture Density Estimation Problem}

Several researchers have viewed clustering as mixture density estimation problem in the framework of probabilistic modeling for cluster analysis. For example, image segmentation may be thought of as a clustering problem. The current literature on statistical image segmentation techniques mostly assumes the image as of containing a mixture of components each of which following normal distribution (Normal or Gaussian Mixture) i.e., $N\left(\mu, \sigma^{2}\right)$ with some weight [6], [13], [14], [15]. In our previous work [1], [2], we assumed the image as of containing a mixture of components each of which following compound normal with gamma distribution (CNGM) i.e., $\operatorname{Normal}\left(\mu, \sigma^{2}\right){ }_{\sigma^{-2}}^{\Lambda} \operatorname{Gamma}\left(c \chi_{v}^{2}\right)$ with some weight. And the whole image is thought of as following the weighted distribution where weighted distribution implies weighted average of the constituent components. In that work, we have studied the feasibility of CNGM vis-a-vis normal mixture (NM) model in consideration to variations of normal distribution.

In this paper, we present the truncated version of compound normal with gamma distribution as a viable model for solving any problem that comes under the scope of cluster analysis. In particular, the scope of this paper is to describe the process of constructing EM algorithm for TCNGM based on he derived update equations obtained in [2] and apply it for image segmentation.

\section{Truncated Compound Normal with Gamma Mixture Model}

The newly derived density function based on Equations (4) and (5) for truncated compound normal with gamma distribution [2] is given as

$f(x)=\frac{2}{c^{1 / 2} B(v / 2,1 / 2)\left[2-\left[I_{a_{1}}\left(\frac{v}{2}, \frac{1}{2}\right)+I_{b_{1}}\left(\frac{v}{2}, \frac{1}{2}\right)\right]\right]}\left[1+\frac{(x-\mu)^{2}}{c}\right]^{-\frac{(v+1)}{2}}$

or

$f(x)=\frac{2}{c^{1 / 2}\left[2 B(v / 2,1 / 2)-\left[B_{a_{1}}\left(\frac{v}{2}, \frac{1}{2}\right)+B_{b_{1}}\left(\frac{v}{2}, \frac{1}{2}\right)\right]\right]}\left[1+\frac{(x-\mu)^{2}}{c}\right]^{-\frac{(v+1)}{2}}$

where $B_{a_{1}}\left(\frac{v}{2}, \frac{1}{2}\right)$ and $B_{b_{1}}\left(\frac{v}{2}, \frac{1}{2}\right)$ are incomplete beta functions. The $f(x)$ in Equation (7) is the new density function for the truncated compound normal with gamma distribution with $a$ and $b$ as left and right truncation points. 


\subsection{Analytical Expressions for Model Parameters, $\alpha_{l}$ and $\theta_{l}\left(\mu_{l}, c_{l}, v_{l}\right)$, For TCNGM}

In [1],[2], the steps involved in the maximum likelihood estimation[16] of the model parameters under Expectation Maximization framework[17] for a mixture density problem have been formally treated in the context of compound normal with gamma mixture model. This has been extended further to define a truncated version, define density function and derive update equations for this[2].In this section, the analytical expressions for the maximum likelihood estimates for model parameters, $\theta_{l}\left(\mu_{l}, c_{l}, v_{l}\right)$ which describe partly the parameter set $\Theta$ are derived in the context of the use of TCNGM under EM framework. EM algorithm optimizes the expected value of the complete data likelihood using expectation and maximization steps iteratively until convergence is reached. This optimization function is formally defined as

$$
\begin{aligned}
& Q\left(\Theta, \Theta^{g}\right)=\sum_{l=1}^{M} \sum_{i=1}^{N} \log \left(\alpha_{l} p_{l}\left(x_{i} \mid \theta_{l}\right)\right) p\left(l \mid x_{i}, \Theta^{g}\right) \\
& =\sum_{l=1}^{M} \sum_{i=1}^{N} \log \left(\alpha_{l}\right) p\left(l \mid x_{i}, \Theta^{g}\right)+\sum_{l=1}^{M} \sum_{i=1}^{N} \log \left(p_{l}\left(x_{i} \mid \theta_{l}\right)\right) p\left(l \mid x_{i}, \Theta^{g}\right)
\end{aligned}
$$

where $\alpha_{l}$ is the prior probability of $l$ th component of the mixture, $p_{l}\left(x_{i} \mid \theta_{l}\right)$ is the conditional probability of $x_{i}$ belonging to $l$ and is defined for our model as in Equation (7), and $p\left(l \mid x_{i}, \Theta^{g}\right)$ is the posterior probability of component $l$ given $x_{i}$ and current estimates of parameters $\Theta^{g}$ and is defined as

$p\left(l \mid x_{i}, \Theta^{g}\right)=\frac{\alpha_{l} p_{l}\left(x_{i} \mid \theta_{l}\right)}{\sum_{l=1}^{M} \alpha_{l} p_{l}\left(x_{i} \mid \theta_{l}\right)}$

A similar treatment is also required for the truncated version except that the modified version of the likelihood function that uses the density function defined as in Equation (7) in the previous section is used. This density function for $l$ th component is nothing but $p_{l}\left(x_{i} \mid \theta_{l}\right)$ that appears in the second term in Equation (8). Hence, in this section, the steps involved for deriving analytical expressions for $\theta_{l}\left(\mu_{l}, c_{l}, v_{l}\right)$ for the truncated version effected by the new density function are only shown.

To maximize Equation (8), we maximize the term containing $\alpha_{l}$ and the term containing $\theta_{l}$ independently since they are not related.

\subsubsection{Analytical Expression For $\alpha_{l}$}

To find the expression for $\alpha_{l}$, we introduce the Lagrange multiplier $\lambda$ (optimization constrained by $\alpha_{l}$ ) with the constraint that $\sum_{l} \alpha_{l}=1$, and solve the following equation as derived in [1],[2]

$\frac{\partial}{\partial \alpha_{l}}\left[\sum_{l=1}^{M} \sum_{i=1}^{N} \log \left(\alpha_{l}\right) p\left(l \mid x_{i}, \Theta^{g}\right)+\lambda\left(\sum_{l} \alpha_{l}-1\right)\right]=0$

Solution of which yields

$\alpha_{l}=\frac{1}{N} \sum_{i=1}^{N} p\left(l \mid x_{i}, \Theta^{g}\right)$

\subsubsection{Analytical Expression For $\theta_{l}\left(\mu_{l}, c_{l}, v_{l}\right)$}

The partial derivatives with respect to the model parameters $\mu_{l}, c_{l}$, and $v_{l}$, after equating them to zero [1], [2], are given as:

$$
\begin{aligned}
& \frac{\partial}{\partial \mu_{l}}\left[\sum_{l=1}^{M} \sum_{i=1}^{N}\left[\log \frac{2}{c_{l}{ }^{1 / 2}\left[2 B\left(\frac{v_{l}}{2}, \frac{1}{2}\right)-\left[B_{a_{1}}\left(\frac{v_{l}}{2}, \frac{1}{2}\right)+B_{b_{1}}\left(\frac{v_{l}}{2}, \frac{1}{2}\right)\right]\right.}\left[1+\frac{\left(x_{i}-\mu_{l}\right)^{2}}{c_{l}}\right]^{-\frac{\left(v_{l}+1\right)}{2}}\right] p\left(l \mid x_{i}, \Theta^{\mathrm{g}}\right)\right]=0 \\
& \frac{\partial}{\partial c_{l}}\left[\sum_{l=1}^{M} \sum_{i=1}^{N}\left[\log \frac{2}{c_{l}^{1 / 2}\left[2 B\left(\frac{v_{l}}{2}, \frac{1}{2}\right)-\left[B_{a_{1}}\left(\frac{v_{l}}{2}, \frac{1}{2}\right)+B_{b_{1}}\left(\frac{v_{l}}{2}, \frac{1}{2}\right)\right]\right]}\left[1+\frac{\left(x_{i}-\mu_{l}\right)^{2}}{c_{l}}\right]^{-\frac{\left(v_{l}+1\right)}{2}}\right] p\left(l \mid x_{i}, \Theta^{\mathrm{g}}\right)\right]=0 \\
& \frac{\partial}{\partial v_{l}}\left[\sum_{l=1}^{M} \sum_{i=1}^{N}\left[\log \frac{2}{c_{l}^{1 / 2}\left[2 B\left(\frac{v_{l}}{2}, \frac{1}{2}\right)-\left[B_{a_{1}}\left(\frac{v_{l}}{2}, \frac{1}{2}\right)+B_{b_{1}}\left(\frac{v_{l}}{2}, \frac{1}{2}\right)\right]\right]}\left[1+\frac{\left(x_{i}-\mu_{l}\right)^{2}}{c_{l}}\right]^{-\frac{\left(v_{l}+1\right)}{2}}\right] p\left(l \mid x_{i}, \Theta^{\mathrm{g}}\right)\right]=0
\end{aligned}
$$


The update equations derived in [2] for $\mu_{l}, c_{l}$, and $v_{l}$ after solving Equations (12), (13), and (14) are given below and these equations are used in the following section to construct Expectation Maximization algorithm.

$$
\begin{aligned}
& \mu_{l}=\frac{\sum_{i=1}^{N} x_{i} p\left(l \mid x_{i}, \Theta^{\mathrm{g}}\right)}{\sum_{i=1}^{N} p\left(l \mid x_{i}, \Theta^{\mathrm{g}}\right)}+\frac{2 c_{l}^{1 / 2}\left[\left[\frac{c_{l}}{c_{l}+\left(a-\mu_{l}\right)^{2}}\right]^{\frac{v_{l}+1}{2}}+\left[\frac{c_{l}}{c_{l}+\left(b-\mu_{l}\right)^{2}}\right]^{\frac{v_{l}+1}{2}}\right]}{\left(v_{l}+1\right) B\left(\frac{v_{l}}{2}, \frac{1}{2}\right)\left[2-\left[I_{a_{1}}\left(\frac{v_{l}}{2}, \frac{1}{2}\right)+I_{b_{1}}\left(\frac{v_{l}}{2}, \frac{1}{2}\right)\right]\right]} \\
& c_{l}=\frac{\left(v_{l}+1\right) \sum_{i=1}^{N}\left(x_{i}-\mu_{l}\right)^{2} p\left(l \mid x_{i}, \Theta^{\mathrm{g}}\right)}{\sum_{i=1}^{N} p\left(l \mid x_{i}, \Theta^{\mathrm{g}}\right)}+\frac{2 c_{l}^{1 / 2}\left[\left(a-\mu_{l}\right)\left[\frac{c_{l}}{c_{l}+\left(a-\mu_{l}\right)^{2}}\right]^{\frac{v_{l}+1}{2}}+\left(b-\mu_{l}\right)\left[\frac{c_{l}}{c_{l}+\left(b-\mu_{l}\right)^{2}}\right]^{\frac{v_{l}+1}{2}}\right]}{B\left(\frac{v_{l}}{2}, \frac{1}{2}\right)\left[2-\left[I_{a_{1}}\left(\frac{v_{l}}{2}, \frac{1}{2}\right)+I_{b_{1}}\left(\frac{v_{l}}{2}, \frac{1}{2}\right)\right]\right]} \\
& v_{l}=\frac{\left[2-\left[I_{a_{1}}\left(\frac{v_{l}}{2}, \frac{3}{2}\right)+I_{b_{1}}\left(\frac{v_{l}}{2}, \frac{3}{2}\right)\right]\right]}{\left[2-\left[I_{a_{1}}\left(\frac{v_{l}}{2}, \frac{1}{2}\right)+I_{b_{1}}\left(\frac{v_{l}}{2}, \frac{1}{2}\right)\right]\right]} \frac{\sum_{i=1}^{N} p\left(l \mid x_{i}, \Theta^{\mathrm{g}}\right)}{\sum_{i=1}^{N} \log \left[1+\frac{\left(x_{i}-\mu_{l}\right)^{2}}{c_{l}}\right] p\left(l \mid x_{i}, \Theta^{\mathrm{g}}\right)}-1
\end{aligned}
$$

\subsection{EM Algorithm for the Proposed Model}

In this section, we describe how image segmentation is performed using EM algorithm for the mixture model defined by truncated compound normal with gamma distribution i.e., $\operatorname{Normal}\left(\mu, \sigma^{2}\right) \underset{\sigma^{-2}}{\Lambda} \operatorname{Gamma}\left(c \chi_{v}^{2}\right)$. The basic steps here are

Step1: Decide $M$, the number of segments based on the number of components of the mixture i.e., fix $\Theta=\left(\alpha_{1}, \ldots, \alpha_{M}, \theta_{1}, \ldots, \theta_{M}\right)$.

/*Here, we decide $M$, the number of segments, by looking at the number of peaks in the histogram of the image data. The decision on $\mathrm{M}$ is highly subjective for natural images. However, it is possible to obtain the number of image regions by optimizing model criterion like minimum description length(MDL)[6].*/

Step2: Initialize $\Theta$.

/*Here, we initialize $\Theta$ using K-means clustering where $\mathrm{K}$ is set to $M$ as decided in Step 1. Since Kmeans gives mean and variance for each $\mathrm{K}$, we use this variance, $\sigma^{2}$, and some initial value for $v_{l}$ to compute initial value for $c_{l}$ by using the relationship between $\sigma^{2}, c$, and, $v$ as derived in [1],[2].*/

Step3: Invoke EM algorithm.

\section{EM Algorithm:}

E-step: Compute the expectation as given by Equation (9)

$p^{(q)}\left(l \mid x_{i}, \Theta^{g}\right)=\frac{\alpha_{l}^{(q)} p_{l}\left(x_{i} \mid \theta_{l}^{(q)}\right)}{\sum_{l=1}^{M} \alpha_{l}^{(q)} p_{l}\left(x_{i} \mid \theta_{l}^{(q)}\right)}(q=0,1,2, \ldots)$

where, according to Equation (7),

$$
p_{l}\left(x_{i} \mid \theta_{l}\right)=\frac{2}{c_{l}^{1 / 2} B\left(v_{l} / 2,1 / 2\right)\left[2-\left[I_{a_{1}}\left(\frac{v_{l}}{2}, \frac{1}{2}\right)+I_{b_{1}}\left(\frac{v_{l}}{2}, \frac{1}{2}\right)\right]\right.}\left[1+\frac{\left(x_{i}-\mu_{l}\right)^{2}}{c_{l}}\right]^{-\frac{\left(v_{l}+1\right)}{2}}
$$

M-step: Compute the updated parameter estimates using Equations (11), (15), (16), and (17) that have been derived in [2].

$$
\alpha_{l}^{(q+1)}=\frac{1}{N} \sum_{i=1}^{N} p^{(q)}\left(l \mid x_{i}, \Theta^{g}\right)
$$




$$
\begin{aligned}
& \mu_{l}^{(q+1)}=\frac{\sum_{i=1}^{N} x_{i} p^{(q)}\left(l \mid x_{i}, \Theta^{g}\right)}{N \alpha_{l}^{(q+1)}}+\frac{2 c_{l}^{(q)^{1 / 2}}\left[\left[\frac{c_{l}^{(q)}}{c_{l}^{(q)}+\left(a-\mu_{l}^{(q)}\right)^{2}}\right]^{\frac{v_{l}^{(q)}+1}{2}}+\left[\frac{c_{l}^{(q)}}{c_{l}^{(q)}+\left(b-\mu_{l}^{(q)}\right)^{2}}\right]^{\frac{v_{l}^{(q)}+1}{2}}\right]}{\left(v_{l}^{(q)}+1\right) B\left(\frac{v_{l}^{(q)}}{2}, \frac{1}{2}\right)\left[2-\left[I_{a_{1}}\left(\frac{v_{l}^{(q)}}{2}, \frac{1}{2}\right)+I_{b_{1}}\left(\frac{v_{l}^{(q)}}{2}, \frac{1}{2}\right)\right]\right]} \\
& v_{l}^{(q+1)}=\frac{\left[2-\left[I_{a_{1}}\left(\frac{v_{l}^{(q)}}{2}, \frac{3}{2}\right)+I_{b_{1}}\left(\frac{v_{l}^{(q)}}{2}, \frac{3}{2}\right)\right]\right]}{\left[2-\left[I_{a_{1}}\left(\frac{v_{l}^{(q)}}{2}, \frac{1}{2}\right)+I_{b_{1}}\left(\frac{v_{l}^{(q)}}{2}, \frac{1}{2}\right)\right]\right]} \frac{N \alpha_{l}^{(q+1)} \sum_{i=1}^{N} \log \left[1+\frac{\left(x_{i}-\mu_{l}^{(q+1)}\right)^{2}}{c_{l}^{(q)}}\right] p^{(q)}\left(l \mid x_{i}, \Theta^{g}\right)}{1} \\
& c_{l}^{(q+1)}=\frac{\left(v_{l}^{(q+1)}+1\right) \sum_{i=1}^{N}\left(x_{i}-\mu_{l}^{(q+1)}\right)^{2} p^{q}\left(l \mid x_{i}, \Theta^{g}\right)}{N \alpha_{l}^{(q+1)}} \\
& +\frac{2 c_{l}^{(q)^{1 / 2}}\left[\left(a-\mu_{l}^{(q)}\right)\left[\frac{c_{l}^{(q)}}{c_{l}^{(q)}+\left(a-\mu_{l}^{(q)}\right)^{2}}\right]^{\frac{v_{l}^{(q)}+1}{2}}+\left(b-\mu_{l}^{(q)}\right)\left[\frac{c_{l}^{(q)}}{c_{l}^{(q)}+\left(b-\mu_{l}^{(q)}\right)^{2}}\right]^{\frac{v_{l}^{(q)}+1}{2}}\right]}{B\left(\frac{v_{l}^{(q)}}{2}, \frac{1}{2}\right)\left[2-\left[I_{a_{1}}\left(\frac{v_{l}^{(q)}}{2}, \frac{1}{2}\right)+I_{b_{1}}\left(\frac{v_{l}^{(q)}}{2}, \frac{1}{2}\right)\right]\right]}
\end{aligned}
$$

$(q=0,1,2, \ldots)$

The stopping criterion is

$\left|\log \mathcal{L}^{(q+1)}-\log \mathcal{L}^{(q)}\right|<\epsilon$

where $\mathcal{L}$ is the likelihood of the parameter estimates, $\epsilon$ is error tolerance. In the above algorithm $a$ and $b$, respectively, are set to 0 and 255 , since these values are considered as left and right truncation points for our truncated version of CNGM.

\subsection{Implementation and Results}

We have implemented the EM algorithm [17],[18] for the truncated version of the proposed compound normal with gamma mixture model in MATLAB and obtained fruitful segmentation results for those images that have been used in [1],[2]. In our experiment, the initialization of the parameters for the specified number of clusters is done using K-means clustering that gives corresponding mean values. Further, here, $c_{l}$ is initialized by using CNGM model characteristics described in [1],[2] with initial value for $v_{l}$ as 3 and the variance computed at the time of initialization by K-means.

We have obtained fruitful segmentation results as shown in Figs.4 through 12. These figures include the original image and its segments, histograms, and probability density plots, respectively, for the truncated version for CNGM (TCNGM) for different $\mathrm{K}$ values. Tables 1 through 9 list the corresponding EM estimates of $\alpha_{l}, \theta_{l}, \log \mathcal{L}$, number of iterations, and segmentation time in seconds.

A close observation of the Figures 4 through 12 and the corresponding MLEs in Tables 1 through 9 shows strong correlation between the respective histograms and the corresponding MLEs and the density plots. For example, for Snake image data given in Fig. 4 and Table 1, the concentration is more around intensity value 130 as shown in its histogram plot. The MLEs for the first mixture component ascertains this fact. For Sunset image data presented in Fig. 5 and Table 2, its histogram has more concentration around 75 and the MLEs for the model parameters for the first component reflects on this. The histogram for Fox image data has more concentration around 10 and 240 which is correlated with MLEs for third and second mixture components respectively that are given in Table 3 . 
For Eagle image data in Fig.6, concentration around 200 in its histogram is reflected by MLEs presented in Table 4 for third component. The histogram for Lady image data has more concentration around 200 followed by 10 and the MLEs for fourth and second mixture components in Table 5 show a similar trend. The histogram for Church image data has more concentration around 100 followed by concentration around 240 which is complemented by the estimates presented in Table 6 for second and third components of the mixture. The histogram for Man image data has more concentration around 100 which is reflected by MLEs given in Table 7 for the fourth component. The histogram for Crane image data shows more concentration around 75 followed by 50 which is reflected by the estimates given in Table 8 for the seventh and second mixture components respectively. The histogram for Horse image data has more concentration around 150 which is reflected by the estimates shown in Table 9 for third component. The respective mixture density plots shown in Figs 4 through 12 also present a similar trend.

The main objective behind the proposed TCNGM model described in [2] is to study its feasibility to solve mixture density estimation problem in the context of truncated data distributions. We understand that image data distributions are well modeled as truncated distributions since the pixel values fall within a finite range.

As we know that gray level image data with 8-bit pixel representation ranges between 0 and 255, the use of TCNGM for image segmentation has been thought to be appropriate. The experimentation of TCNGM under EM framework using MATLAB presented here has produced strong correlations between characteristic plots and EM estimates for the chosen image datasets. The analysis of the results described above strongly supports this tendency.

Table1. TCNGM ML Estimates for Snake Data $(K=2)$

\begin{tabular}{|c|c|c|c|c|c|c|c|}
\hline Image & \multirow{2}{*}{$\alpha_{l}$} & \multicolumn{3}{|c|}{$\theta_{l}$} & $\log \mathcal{L}$ & $\begin{array}{c}\text { No. of } \\
\text { Iterations }\end{array}$ & $\begin{array}{c}\text { Segmentation } \\
\text { Time(Seconds) }\end{array}$ \\
\cline { 3 - 5 } Snake & 0.9381 & 130.6596 & 3673.7409 & 72.2379 & & -35023.6902 \\
$(\mathrm{~K}=2)$ & 26 & 866.3281 \\
\cline { 2 - 5 } & 0.0619 & 77.7117 & 9605.9975 & 62.1075 & & \\
\hline
\end{tabular}

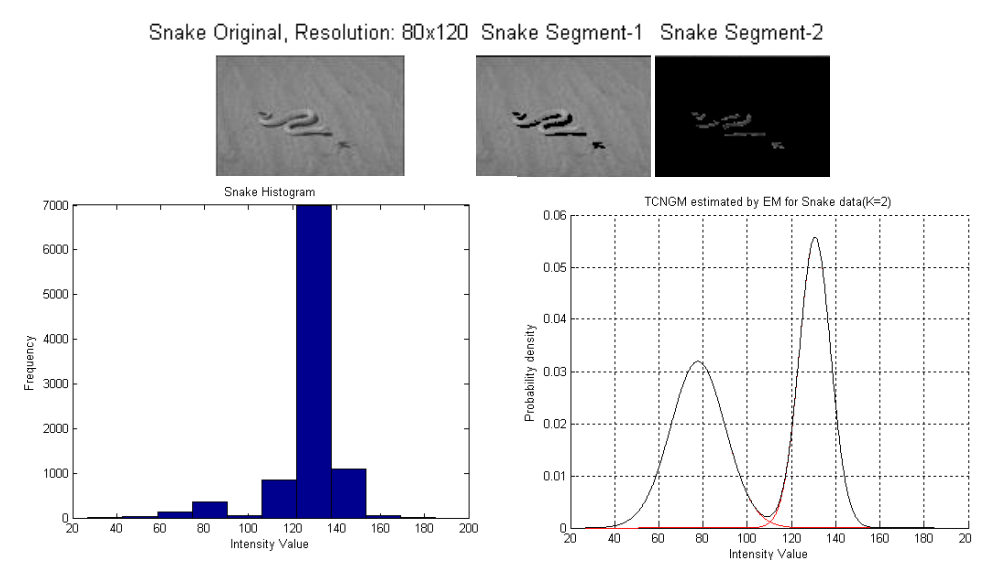

Fig4 Snake Data. Original Image and its segments (above), Histogram and Mixture Density Plot (below)

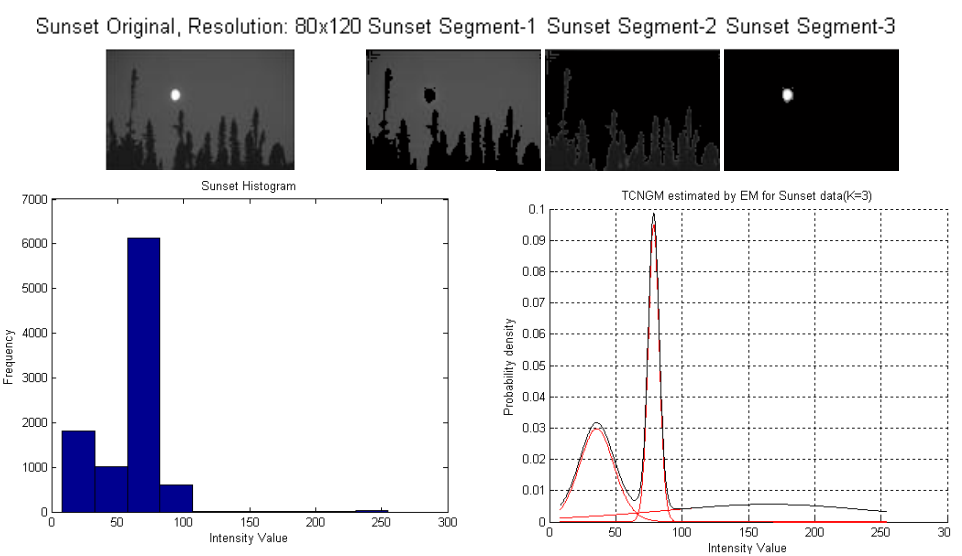

Fig5 Sunset Data. Original Image and its segments (above), Histogram and Mixture Density Plot (below) 
Image Segmentation Using Truncated Compound Normal with Gamma Mixture Model

Table2. TCNGM ML Estimates for Sunset Data $(k=3)$

\begin{tabular}{|c|c|c|c|c|c|c|c|}
\hline Image & \multirow[t]{2}{*}{$\alpha_{l}$} & \multicolumn{3}{|c|}{$\theta_{l}$} & \multirow[t]{2}{*}{$\log \mathcal{L}$} & \multirow{2}{*}{$\begin{array}{c}\text { No. of } \\
\text { Iterations }\end{array}$} & \multirow{2}{*}{$\begin{array}{l}\text { Segmentation } \\
\text { Time(Seconds) }\end{array}$} \\
\hline \multirow{4}{*}{ Sunset } & & $\mu_{l}$ & $c_{l}$ & $v_{l}$ & & & \\
\hline & 0.6692 & 78.6252 & 400.4247 & 23.1710 & \multirow{3}{*}{$\begin{array}{c}-37102.2624 \\
(K=3)\end{array}$} & \multirow{3}{*}{9} & \multirow{3}{*}{531.3750} \\
\hline & 0.3242 & 35.5839 & 2410.8514 & 13.7319 & & & \\
\hline & 0.0066 & 165.9272 & 43587.8925 & 5.5880 & & & \\
\hline
\end{tabular}

Table3. TCNGM ML Estimates for Fox Data $(K=3)$

\begin{tabular}{|c|c|c|c|c|c|c|c|}
\hline Image & \multirow[t]{2}{*}{$\alpha_{l}$} & \multicolumn{3}{|c|}{$\theta_{l}$} & \multirow[t]{2}{*}{$\log \mathcal{L}$} & \multirow{2}{*}{$\begin{array}{c}\text { No. of } \\
\text { Iterations }\end{array}$} & \multirow{2}{*}{$\begin{array}{l}\text { Segmentation } \\
\text { Time(Seconds) }\end{array}$} \\
\hline \multirow{4}{*}{ Fox } & & $\mu_{l}$ & $c_{l}$ & $v_{l}$ & & & \\
\hline & 0.0886 & 116.0769 & 33501.1298 & 6.0447 & \multirow{3}{*}{$\begin{array}{c}-36632.3612 \\
(K=3)\end{array}$} & \multirow{3}{*}{9} & \multirow{3}{*}{493.5469} \\
\hline & 0.3303 & 253.0665 & 204.1824 & 17.7048 & & & \\
\hline & 0.5811 & 13.9604 & 517.3162 & 20.7158 & & & \\
\hline
\end{tabular}

Fox Original, Resolution: $80 \times 120 \quad$ Fox Segment-1 $\quad$ Fox Segment-2 $\quad$ Fox Segment-3
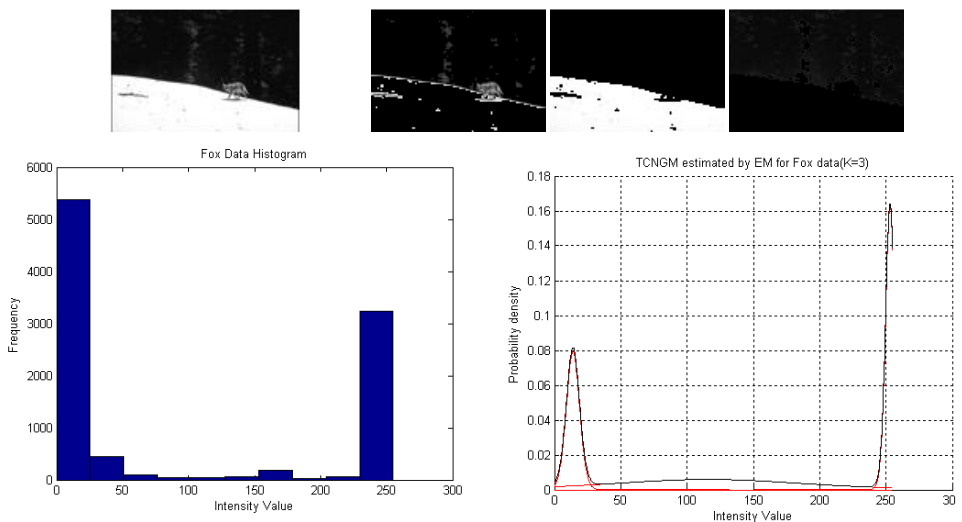

Fig6 Fox Data. Original Image and its segments (above), Histogram and Mixture Density Plot (below)

Table4. TCNGM ML Estimates for Eagle Data $(K=4)$

\begin{tabular}{|c|c|c|c|c|c|c|c|}
\hline Image & \multirow[t]{2}{*}{$\alpha_{l}$} & \multicolumn{3}{|c|}{$\theta_{l}$} & \multirow[t]{2}{*}{$\log \mathcal{L}$} & \multirow{2}{*}{$\begin{array}{c}\text { No. of } \\
\text { Iterations }\end{array}$} & \multirow{2}{*}{$\begin{array}{l}\text { Segmentation } \\
\text { Time(Seconds) }\end{array}$} \\
\hline \multirow{5}{*}{ Eagle } & & $\mu_{l}$ & $c_{l}$ & $v_{l}$ & & & \\
\hline & 0.1089 & 160.9267 & 3922.3254 & 9.2782 & \multirow{4}{*}{$\begin{array}{c}-44456.4675 \\
(K=4)\end{array}$} & \multirow{4}{*}{4} & \multirow{4}{*}{295.3438} \\
\hline & 0.1203 & 111.4112 & 6938.3657 & 8.9274 & & & \\
\hline & 0.6446 & 195.2787 & 603.0092 & 10.6518 & & & \\
\hline & 0.1089 & 160.9267 & 3922.3254 & 9.2782 & & & \\
\hline
\end{tabular}

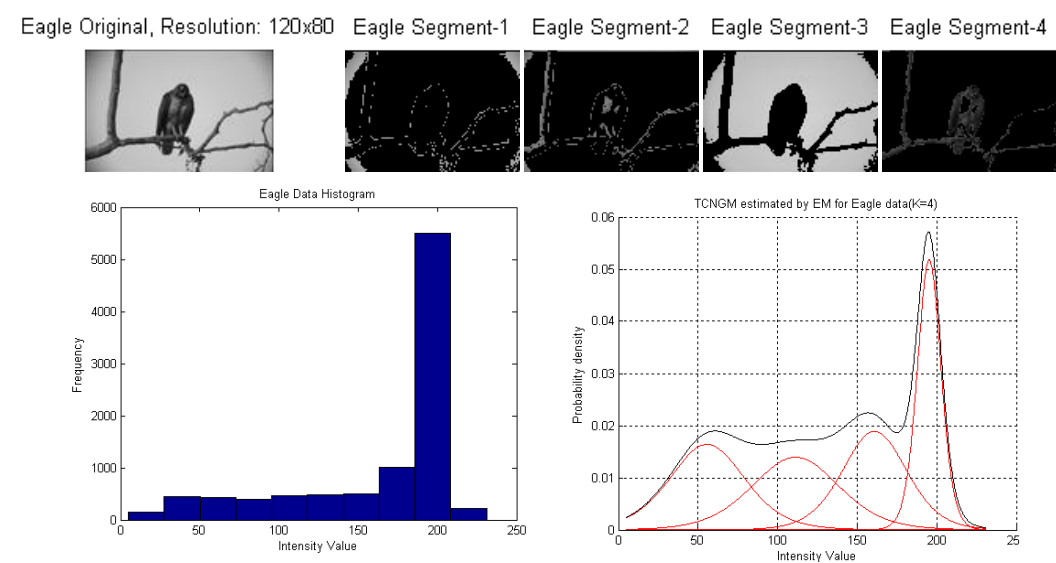

Fig7 Eagle Data. Original Image and its segments (above), Histogram and Mixture Density Plot (below) 
Viziananda Row Sanapala et al.

Table5. TCNGM ML Estimates for Lady Data $(K=4)$

\begin{tabular}{|c|c|c|c|c|c|c|c|}
\hline Image & $\alpha_{l}$ & \multicolumn{3}{|c|}{$\theta_{l}$} & $\log \mathcal{L}$ & $\begin{array}{c}\text { No. of } \\
\text { Iterations }\end{array}$ & $\begin{array}{c}\text { Segmentation } \\
\text { Time(Seconds) }\end{array}$ \\
\cline { 3 - 6 } & & $\mu_{l}$ & $c_{l}$ & $v_{l}$ & & \\
\cline { 2 - 5 } Lady & 0.2372 & 130.9775 & 36563.1525 & 23.8566 & & 1300.641 \\
\cline { 2 - 5 } & 0.2180 & 14.5879 & 662.7173 & 12.8592 & -50330.2265 & 17 & \\
\cline { 2 - 5 } & 0.2341 & 99.4459 & 25535.1006 & 12.6374 & & & \\
\cline { 2 - 5 } & 0.3107 & 196.0037 & 3827.2277 & 28.6799 & & & \\
\hline
\end{tabular}
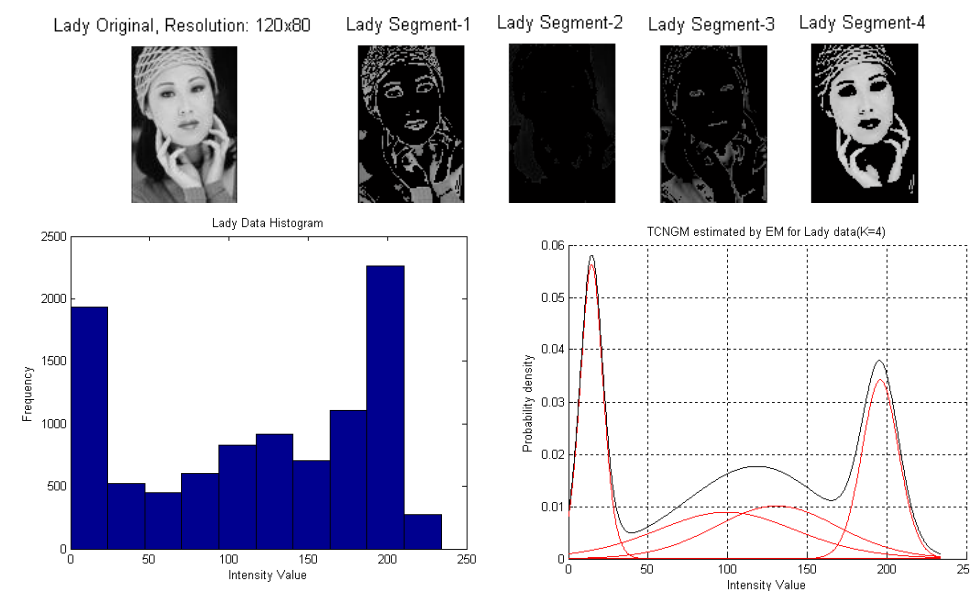

Fig8 Lady Data. Original Image and its segments (above), Histogram and Mixture Density Plot (below)

Table6. TCNGM ML Estimates for Church Data $(K=5)$

\begin{tabular}{|c|c|c|c|c|c|c|c|}
\hline Image & \multirow{2}{*}{$\alpha_{l}$} & \multicolumn{3}{|c|}{$\theta_{l}$} & $\log \mathcal{L}$ & $\begin{array}{c}\text { No. of } \\
\text { Iterations }\end{array}$ & $\begin{array}{c}\text { Segmentation } \\
\text { Time(Seconds) }\end{array}$ \\
\cline { 3 - 5 } & 0.0242 & 40.8355 & 560.9751 & 10.1860 & & \\
\cline { 2 - 5 } Church & 0.4780 & 92.6533 & 851.9156 & 8.8252 & & \\
\cline { 2 - 5 } & 0.3174 & 246.0361 & 692.6319 & 7.6565 & -46073.7706 & 3 & 300.8594 \\
\cline { 2 - 5 } & 0.0682 & 185.3611 & 5247.4521 & 6.6640 & & \\
\cline { 2 - 5 } & 0.1122 & 135.8699 & 4479.3548 & 7.4084 & & & \\
\hline
\end{tabular}

Church Original, Resolution:80x120 Church Segment-1 Church Segment-2 Church Segment-3 Church Segment-4 Church Segment-5
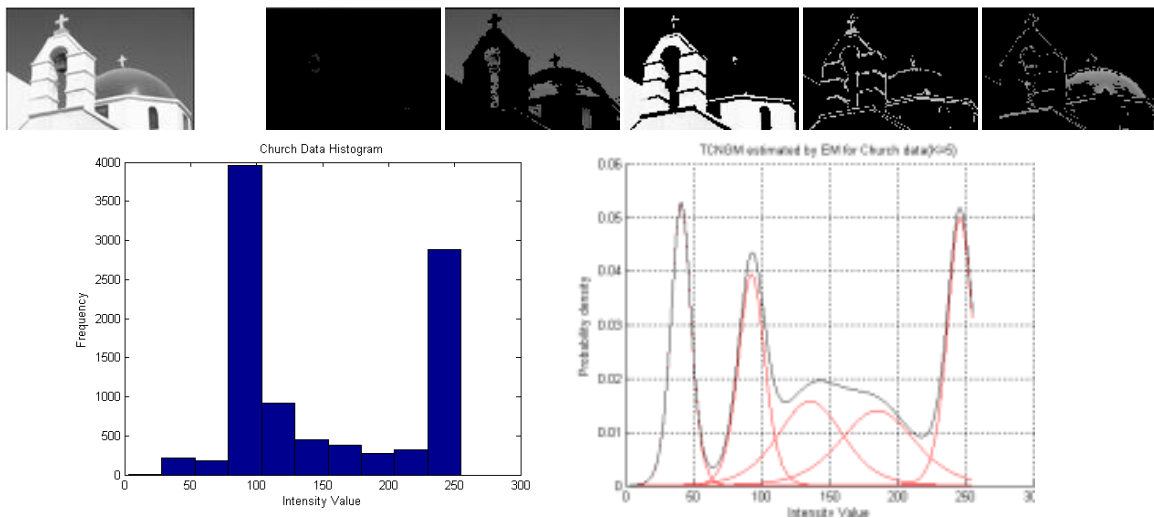

Fig9 Church Data. Original Image and its segments (above), Histogram and Mixture Density Plot (below)

Table7. TCNGM ML Estimates for Man Data $(K=6)$

\begin{tabular}{|c|c|c|c|c|c|c|c|}
\hline Image & \multirow[t]{2}{*}{$\alpha_{l}$} & \multicolumn{3}{|c|}{$\theta_{l}$} & \multirow[t]{2}{*}{$\log \mathcal{L}$} & \multirow{2}{*}{$\begin{array}{c}\text { No. of } \\
\text { Iterations }\end{array}$} & \multirow{2}{*}{$\begin{array}{c}\text { Segmentation } \\
\text { Time(Seconds) }\end{array}$} \\
\hline \multirow{7}{*}{ Man } & & $\mu_{l}$ & $c_{l}$ & $v_{l}$ & & & \\
\hline & 0.1568 & 51.1117 & 4511.8358 & 36.5965 & \multirow{6}{*}{$\begin{array}{c}-46812.1734 \\
(K=6)\end{array}$} & \multirow{6}{*}{22} & \multirow{6}{*}{2394.6094} \\
\hline & 0.2128 & 104.0818 & 5213.8420 & 40.1993 & & & \\
\hline & 0.0994 & 67.0034 & 10259.8758 & 35.7816 & & & \\
\hline & 0.3328 & 107.1731 & 4078.2365 & 40.7203 & & & \\
\hline & 0.0454 & 127.2467 & 6914.7080 & 37.2054 & & & \\
\hline & 0.1528 & 175.8975 & 60375.5438 & 9.7511 & & & \\
\hline
\end{tabular}




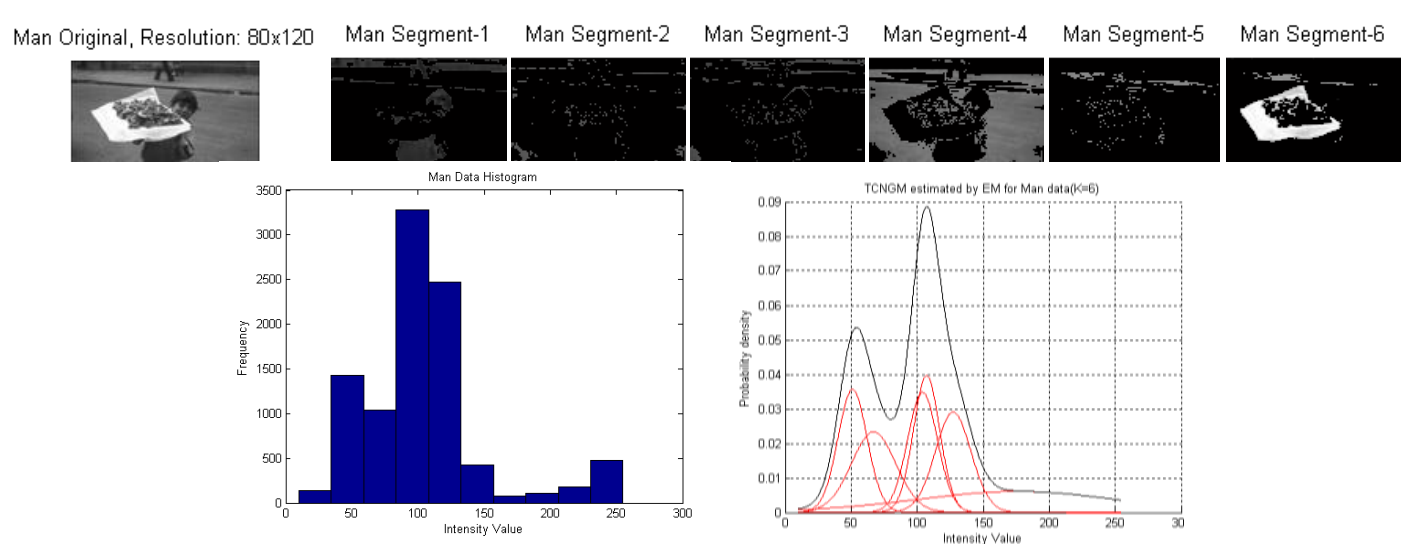

Fig10 Man Data. Original Image and its segments (above), Histogram and Mixture Density Plot (below)

Table8. TCNGM ML Estimates for Crane Data $(K=7)$

\begin{tabular}{|c|c|c|c|c|c|c|c|}
\hline Image & \multirow[t]{2}{*}{$\alpha_{l}$} & \multicolumn{3}{|c|}{$\theta_{l}$} & \multirow[t]{2}{*}{$\log \mathcal{L}$} & \multirow{2}{*}{$\begin{array}{c}\text { No. of } \\
\text { Iterations }\end{array}$} & \multirow{2}{*}{$\begin{array}{l}\text { Segmentation } \\
\text { Time(Seconds) }\end{array}$} \\
\hline \multirow{8}{*}{ Crane } & & $\mu_{l}$ & $c_{l}$ & $v_{l}$ & & & \\
\hline & 0.0729 & 119.5908 & 12838.0214 & 17.0312 & \multirow{7}{*}{$\begin{array}{c}-46840.0419 \\
(K=7)\end{array}$} & \multirow{7}{*}{9} & \multirow{7}{*}{1079.5313} \\
\hline & 0.2266 & 60.6184 & 2696.5310 & 16.9804 & & & \\
\hline & 0.0929 & 174.5387 & 1195.2051 & 22.8335 & & & \\
\hline & 0.0357 & 244.9591 & 4116.7951 & 12.4592 & & & \\
\hline & 0.1596 & 92.8795 & 5036.9914 & 17.5185 & & & \\
\hline & 0.1626 & 48.0420 & 1856.1957 & 17.4924 & & & \\
\hline & 0.2497 & 75.5196 & 2790.3153 & 17.5613 & & & \\
\hline
\end{tabular}

Crane Original, Resolution: $120 \times 80$ Crane Segment- 1 Crane Segment- 2 Crane Segment-3 Crane Segment-4 Crane Segment- 5 Crane Segment- 6 Crane Segment- 7
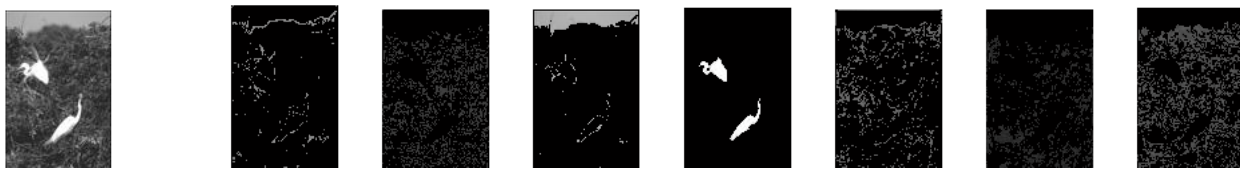

(a)
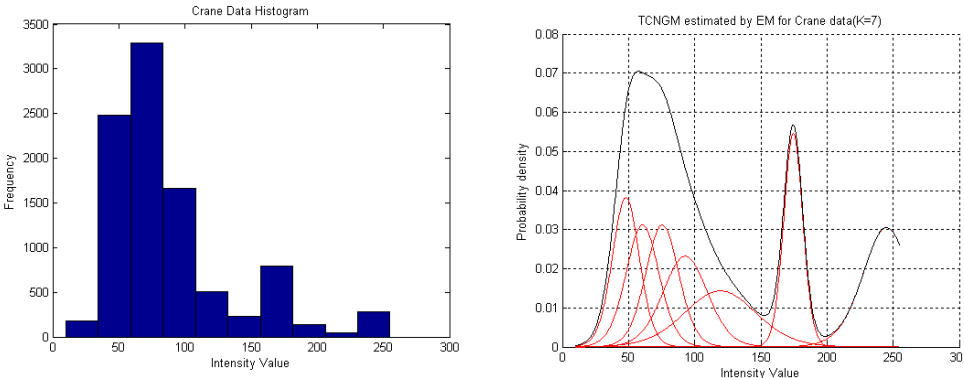

Fig11 Crane Data. Original Image and its segments (above), Histogram and Mixture Density Plot (below)

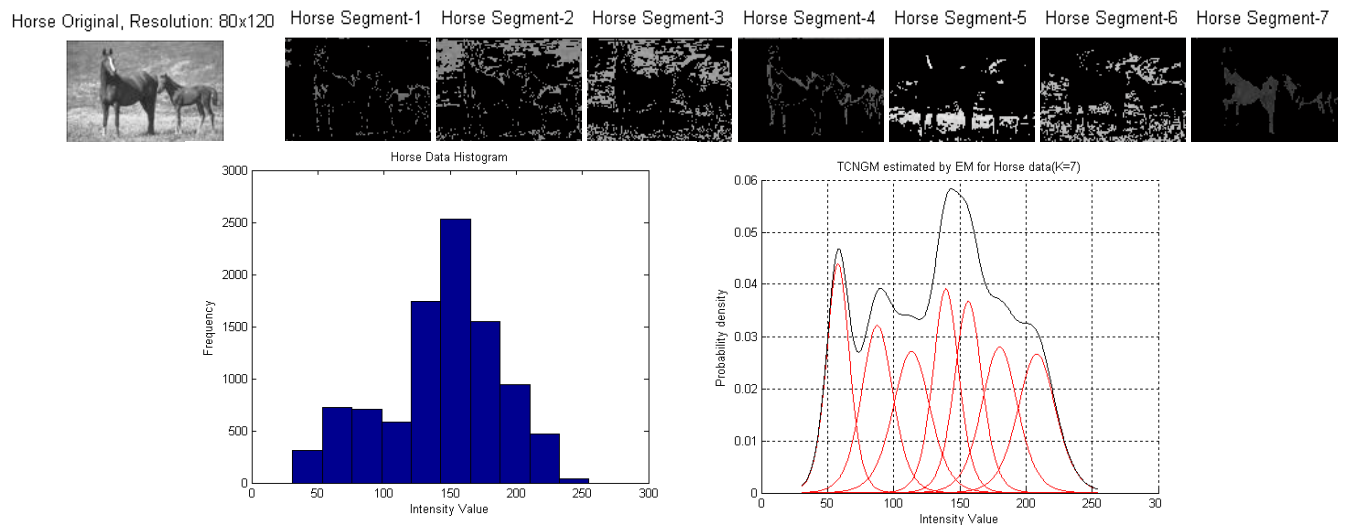

Fig12 Horse Data. Original Image and its segments (above), Histogram and Mixture Density Plot (below) 
Viziananda Row Sanapala et al.

Table9. TCNGM ML Estimates for Horse Data $(K=7)$

\begin{tabular}{|c|c|c|c|c|c|c|c|}
\hline Image & \multirow[t]{2}{*}{$\alpha_{l}$} & \multicolumn{3}{|c|}{$\theta_{l}$} & \multirow[t]{2}{*}{$\log \mathcal{L}$} & \multirow{2}{*}{$\begin{array}{l}\text { No. of } \\
\text { Iterations }\end{array}$} & \multirow{2}{*}{$\begin{array}{l}\text { Segmentation } \\
\text { Time(Seconds) }\end{array}$} \\
\hline \multirow{8}{*}{ Horse } & & $\mu_{l}$ & $c_{l}$ & $v_{l}$ & & & \\
\hline & 0.0699 & 113.5461 & 1604.9862 & 7.9121 & \multirow{7}{*}{$\begin{array}{c}-48815.3291 \\
(K=7)\end{array}$} & \multirow{7}{*}{3} & \multirow{7}{*}{450.7344} \\
\hline & 0.2182 & 139.4819 & 804.7983 & 8.2259 & & & \\
\hline & 0.2365 & 156.3763 & 893.9370 & 8.0697 & & & \\
\hline & 0.0872 & 87.5816 & 1160.9240 & 8.0030 & & & \\
\hline & 0.1062 & 208.1673 & 1637.6123 & 7.7005 & & & \\
\hline & 0.1825 & 180.0689 & 1507.3609 & 7.8942 & & & \\
\hline & 0.0995 & 57.9416 & 627.8301 & 8.1048 & & & \\
\hline
\end{tabular}

\section{Conclusions}

In this paper, we have presented the experimental details of the work we carried out in [2] for the proposed truncated version of the compound normal with gamma mixture model (TCNGM) and its incorporation into EM framework. The TCNGM has been found to produce fruitful results in terms of its convergence performance. The feasibility of the model for image segmentation has been thoroughly examined. Its segmentation quality performance vis-a-vis other methods will be considered later as an extension to this work.

\section{ACKNOWLEDGMENTS}

The authors hereby express their sincere gratitude to Andhra University, Visakhapatnam, India where they have completed more than twenty five years of fruitful teaching service and thank the institution for having given them an opportunity to work in a conducive academic and research environment.

\section{REFERENCES}

[1] Viziananda Row Sanapala, Sreenivasa Rao Kraleti, and Srinivasa Rao Peri. Image Segmentation Using Compound Normal with Gamma Mixture Model, International Journal of Computer Science Issues, Volume 12, Issue 4, July 2015, ISSN (Print): 1694-0814 | ISSN (Online): 16940784, www.IJCSI.org.

[2] S. Viziananda Row, Image Segmentation Using Compound Normal with Gamma Mixture Model and its Truncated Version, Ph. D. Thesis, Andhra University, Visakhapatnam, India, 2016.

[3] J. Canny. A computational approach to edge detection, IEEE Trans. Pattern Anal. Mach. Intell., vol. 8, no. 6, pp. 679-698, 1986.

[4] Paul Bao, Lei Zhang, and Xiaolin Wu. Canny Edge Detection Enhancement by Scale Multiplication, IEEE Transactions On Pattern Analysis And Machine Intelligence, vol. 27, no. 9, pp.1485-1490, 2005.

[5] Yang Zhang and Peter. I Rockett. The Bayesian Operating Point of the Canny Edge Detector, IEEE Transactions On Image Processing, vol. 15, no. 11, pp. 3409-3416, 2006

[6] T. Lei and J. K. Udupa. Performance evaluation of finite normal mixture model-based image segmentaion techniques. IEEE Transactions on Image Processing, vol 12, no 10, pp. 1153-1169, 2003.

[7] R. Unnikrishnan, C. Pantofaru, and M. Hebert. Toward objective evaluation of image segmentation algorithms", IEEE Transactions on Pattern Analysis and Machine Intelligence, vol 29, no 6, pp. 929-944, 2007.

[8] Margaret H. Dunham. Data Mining: Introductory and Advanced Topics, Low Price Edition, Pearson Education, India.

[9] J. K. Udupa and S. Samarasekera. Fuzzy connectedness and object definition: Theory, algorithms, and applications in image segmentation, GraphModels Image Process., vol. 38, no. 3, pp. 246-261, 1996.

[10] Alexander M. Mood, Franklin A. Graybill, and Duane C. Boes. Introduction to the theory of Statistics, Tata McGraw-Hill, Third Edition, 2001.

[11] S. C. Gupta and V. K. Kapoor. Fundamentals of Mathematical Statistics, Sultan Chand and Sons, New Delhi, Eleventh Edition, 2002.

[12] Normal L. Johnson, Samuel Kotz and N. Balakrishnan. Continuous Univariate Distributions, Vol-I, Second Edition. John Wiley\&Sons, 2007. 
[13] J. Zhang and J. M. Modestino. A model fitting approach to cluster validity with application to stochastic model based image segmentation, IEEE Transactions on Pattern Analysis and Machine Intelligence, vol. 12, no. 10, pp.1009-1016, 1990.

[14] T. Lei and W. Sewchand. Statistical approach to X-ray CT imaging and its applications in image analysis-Part 2: A new stochastic model based image segmentation technique for CT image, IEEE Transactions on Medical Imaging, vol. 11, no. 1, pp. 62-69, 1992.

[15] Z. Liang and J. R. MacFall. "Parameter estimation and tissue segmentation of multispectral MR images." IEEE Transactions on Medical Imaging, vol. 13, no.3, pp. 441-449, 1994.

[16] A. P. Dempster, N. M. Laird and D. B. Rubin. Maximum-likelihood from incomplete data via the EM algorithm, J. Royal Statist. Soc. Ser. B (methodological), 39 (1977), pp. 1-38.

[17] J. A. Bilmes. "A gentle tutorial of the EM algorithm and its application to parameter estimation for Gaussian mixture and hidden Markov models." International Computer Science Institute, Berkely CA, 94704, pp 1-13.

[18] MATLAB open source code for EM algorithm for Gaussian mixture model written by Patrick P C Tsui, PAMI research group, University of Waterloo, 2006.

\section{AUTHORS' BIOGRAPHY}

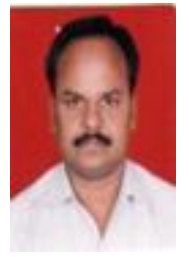

Viziananda Row Sanapala, received his B.Tech in Chemical Engg in 1983, M Tech in Computer Science \& Technology in 1987, and Ph D in Computer Science in 2016 all from Andhra University. He is currently Associate Professor in Computer Science \& Systems Engg in Andhra University, India. His research interests include image processing, pattern recognition, and others.

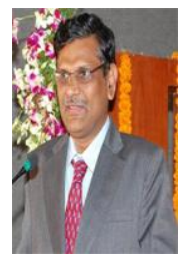

Sreenivasa Rao Kraleti, received his M Sc in Statistics in 1982 and Ph D in Statistics in 1990 both from Andhra University. He is currently serving as Professor of Statistics in Andhra University, India. His research interests are data analysis, stochastic modeling

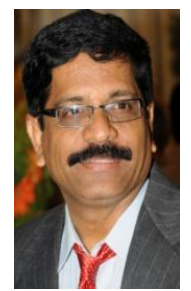

Srinivasa Rao Peri, received his M Sc in Statistics in 1982 from Andhra University and $\mathrm{Ph} \mathrm{D}$ in Computer Science in 1987 from IIT, Kharagpur, India. He is serving as Professor in Computer Science \& Systems Engg in Andhra University, India. His research interests include image processing, queueing theory, and others 